\title{
Mir-302 reprograms human skin cancer cells into a pluripotent ES-cell-like state
}

\author{
SHI-LUNG LIN, ${ }^{1}$ DONALD C. CHANG, ${ }^{1}$ SAMANTHA CHANG-LIN, ${ }^{1}$ CHUN-HUNG LIN, ${ }^{2}$ DAVID T.S. WU, ${ }^{3}$ \\ DAVID T. CHEN, ${ }^{3}$ and SHAO-YAO YING ${ }^{1}$ \\ ${ }^{1}$ Department of Cell and Neurobiology, Keck School of Medicine, University of Southern California, Los Angeles, California 90033, USA \\ ${ }^{2}$ Dental Department, Taiwan Adventist Hospital, Taipei City 10556, Taiwan, Republic of China \\ ${ }^{3}$ Department of Ear, Nose and Throat, Tzu Chi General Hospital, Hualien 970, Taiwan, Republic of China
}

\begin{abstract}
Renewal of stem cells differs from cancer cell growth in self-controlled cell division. The mir-302 microRNA (miRNA) family (mir-302s) is expressed most abundantly in slow-growing human embryonic stem (ES) cells, and quickly decreases after cell differentiation and proliferation. Therefore, mir-302s was investigated as one of the key factors essential for maintenance of ES cell renewal and pluripotency in this study. The Pol-II-based intronic miRNA expression system was used to transgenically transfect the mir-302s into several human cancer cell lines. The mir-302 - transfected cells, namely, miRNA-induced pluripotent stem (mirPS) cells, not only expressed many key ES cell markers, such as Oct3/4, SSEA-3, SSEA-4, Sox2, and Nanog, but also had a highly demethylated genome similar to a reprogrammed zygotic genome. Microarray analyses further revealed that genomewide gene expression patterns between the mirPS and human ES $\mathrm{H} 1$ and $\mathrm{H} 9$ cells shared over $86 \%$ similarity. Using molecular guidance in vitro, these mirPS cells could differentiate into distinct tissue cell types, such as neuron-, chondrocyte-, fibroblast-, and spermatogonia-like primordial cells. Based on these findings, we conclude that mir-302s not only function to reprogram cancer cells into an ES-like pluripotent state but also to maintain this state under a feeder-free cultural condition, which may offer a great opportunity for therapeutic intervention.
\end{abstract}

Keywords: miRNA; mir-302; embryonic stem cell; induced pluripotent stem cell; stem cell generation; renewal; pluripotency; embryoid body; feeder-free cell culture; epigenetic reprogramming; cell differentiation

\section{INTRODUCTION}

The concept of cancer stem cells indicates that transformed stem cells within a tumor are able to self-renew and differentiate into a heterogeneous tumor population (Reya et al. 2001). However, there is no clear mechanism underlying such stem cell-cancer cell transformation or vice versa. In the clinic, it is very frequently observed that cancer

Reprint requests to: Shi-Lung Lin, Department of Cell and Neurobiology, Keck School of Medicine, BMT-403, University of Southern California, 1333 San Pablo Street, Los Angeles, CA 90033; e-mail: lins@ usc.edu; fax: (323) 442-3466; or Shao-Yao Ying, Department of Cell and Neurobiology, Keck School of Medicine, BMT-403, University of Southern California, 1333 San Pablo Street, Los Angeles, CA 90033; e-mail: sying@usc.edu; fax: (323) 442-3466.

Abbreviations: miRNA, microRNA; mir-302s, the mir-302 family; ES, embryonic stem; iPS, induced pluripotent stem; mirPS, mir-302-induced pluripotent stem; EB, embryoid body; CSC, cancerous stem cell; Pol-II, RNA polymerase type-II; NMD, nonsense-mediated decay.

Article published online ahead of print. Article and publication date are at http://www.rnajournal.org/cgi/doi/10.1261/rna.1162708. progression is generally associated with poor differentiation (high grade) of human tumor cells. Recent findings have also shown that poorly differentiated tumors preferentially overexpress genes normally enriched in human embryonic stem (ES) cells, such as targets of Oct3/4, Sox2, and Nanog transcription factors. Nevertheless, the concurrent expression of these transcription factors themselves is not often detected in the poorly differentiated tumors (Ben-Porath et al. 2008). It is conceivable that a different set of transcriptional regulators in place of Oct3/4, Sox2, and Nanog may function in poorly differentiated tumor cells to promote their "stemness" signatures. Therefore, finding the way by which stem cells substitute for these cancer-related transcriptional regulators may lead to breakthroughs in both cancer therapy and stem cell generation. To this end, we report here that the present study of mir-302 function provides the first insight into the mechanism underlying reverse transformation of human cancer cells into ES-like pluripotent cells.

The mir-302 family (mir-302s) consists of four highly homologous microRNA (miRNA) members, which are 
transcribed together as a noncoding RNA cluster containing mir-302b, mir-302c, mir-302a, mir-302d, and mir-367 in a $5^{\prime}$-to-3' direction (Suh et al. 2004). They are expressed most abundantly in slow-growing human ES cells and quickly decrease after cell differentiation and proliferation (Suh et al. 2004). Given that miRNAs are characterized as small inhibitory RNAs capable of suppressing the translation of target genes with high complementarity (Bartel 2004), mir-302s is a likely candidate zygotic inhibitor of premature cell differentiation during early embryonic development. As shown in the miRBase::Sequences program (http://microrna.sanger.ac.uk/sequences/), mir-302s can target over 445 human genes, and most of these targets are developmental signals involving the initiation and/or facilitation of lineage-specific cell differentiation during early human embryogenesis. These target genes are listed in the target prediction sites linked to the miRBase::Sequences program at the Sanger website, including TARGETSCAN (http://www.targetscan.org/vert_42/) and PICTAR-VERT (http://pictar.bio.nyu.edu/cgi-bin/PicTar_vertebrate.cgi?). Thus, we hypothesize that mir-302s are key factors essential for ES cell maintenance, possibly able to reprogram cancer cells into a more ES-cell-like state.

To test the function of mir-302s, we developed a retroviral Pol-II-based intronic miRNA expression system, namely, $p L N C X 2-r T-S p R N A i$ (Fig. 1), and successfully used it to generate several transgenic miRNA-expressing cell lines and animals (Lin and Ying 2006; Lin et al. 2006). The same transgenic approach has also been used to generate gene-knockout mice for human disease research (Xia et al. 2006). Intronic miRNA expression is a prevalent event in mammals because $\sim 50 \%$ of mammalian miRNAs are encoded within the introns of protein-coding genes (Rodriguez et al. 2004). These miRNAs are transcribed by type-II RNA polymerases (Pol-II) and excised by spliceosomes and other RNase III endonucleases to form mature miRNAs (Danin-Kreiselman et al. 2003; Lin et al. 2003). However, Drosha may not be required for this process (Ruby et al. 2007). The composition of this mir-302expressing $p L N C X 2-r T-S p R N A i$ vector is shown in Figure $2 \mathrm{~A}$. Using this vector-based transfection strategy, we have generated two mir-302-expressing mirPS cell lines, namely, mirPS-Colo and mirPS-PC3, derived from human melanoma Colo and prostate cancer PC3 cells, respectively, and confirmed that mirPS-Colo cells highly resembled human ES H1 and H9 cells in stem cell renewal and pluripotency.

\section{RESULTS}

\section{Generation of human ES-like mir-302-induced pluripotent stem (mirPS) cell lines and embryoid bodies}

After the $p L N C X 2-r T-S p R N A i$ retroviral transfection with a predesigned mir-302 pre-miRNA cluster transgene (Fig.
2B), $\sim 95 \%-98 \%$ of the transfected cells underwent apoptosis with the remaining $2 \%-5 \%$ of the cells transformed into ES-like mirPS cells. The transfection rates of mir-302s into Colo and PC3 cells were $99.8 \%$ and $99.4 \%$, respectively, as determined by FACS flow cytometry sorting with mir-302 maker RGFP and ES marker Oct3/4 antibodies (Fig. 2C). These mirPS cells could grow in either DMEM/ F12 or RPMI 1640/B27 medium supplemented with 10\% charcoal-stripped FBS, $4 \mathrm{mM}$ L-glutamine, $1 \mathrm{mM}$ sodium pyruvate, $5 \mathrm{ng} / \mathrm{mL}$ activin, $5 \mathrm{ng} / \mathrm{mL}$ noggin, $3 \mathrm{ng} / \mathrm{mL} \mathrm{bFGF}$, and an equal mixture of $0.5 \mu \mathrm{M}$ Y-27632 and $0.5 \mu \mathrm{M}$ GSK3 inhibitor $\mathrm{XV}$, at $37^{\circ} \mathrm{C}$ under $5 \% \mathrm{CO}_{2}$. Under this feederfree cultural condition, the average cell cycle of the mirPS cells was $\sim 20-24 \mathrm{~h}$, indicating a very slow cell renewal rate compared with their cancerous counterparts $(\sim 4-6 \mathrm{~h}$ per cell cycle).

Flow cytometry analysis comparing DNA content to cell cycle stages showed a greater than $67 \%$ reduction in the mirPS mitotic cell population (Fig. 2D). The mitotic cell population (M phase) was decreased from $36.5 \%$ to $11.5 \%$ in mirPS-Colo and from $38.4 \%$ to $12.6 \%$ in mirPS-PC3 cells, whereas no change was found in the control cells transfected with either an empty $p L N C X 2-r T-S p R N A i$ vector (cell+vector) or a vector encoding an off-target mir-gfp pre-miRNA construct (cell+mir-gfp). However, transfection of a vector encoding mutated mir-302s abolished the effects of mir-302s on cell cycle suppression and Oct3/4 gene activation (Supplemental Fig. 1). The mutated mir$302 \mathrm{~s}$ were formed by replacing the first eight nucleotides of the mir-302 seed sequence (UAAGUGCU) with AUUAA UUA in all four isoforms. Accordingly, the mirPS cell morphology (lower panels) was changed from a spindle- or asterisk-like form to a more rounded shape, indicating that the mirPS cells may have lost their ability to migrate. As shown in Figure 2E, metastatic PC3 cells quickly migrated over time, whereas mirPS-PC3 cells remained stationary. No morphological change was observed in all other controls. Thus, such transgenic mir-302s expression is sufficient to transform human cancer cells into a more ES-like cell morphology and rate of cell division, suggesting a very beneficial use in cancer therapy.

MirPS cells were able to form compact colonies reminiscent of embryoid bodies (EBs) derived from human ES cells (Fig. 2F; Supplemental Fig. 2). When dissociated with collagenase IV and then cultivated in RPMI 1640 medium supplemented with $10 \%$ FBS, but without GSK and ROCK/ Ras inhibitors, many of these EB-like cells differentiated into neuronal cells based on the presence of positive neuronal markers Tuj1 and ABCA2. We further noted that mirPS-PC3 EB cells could only differentiate into neuronal cell types, while mirPS-Colo EB cells formed teratoma-like primordial tissue cysts in immunocompromised SCIDbeige mice (Fig. 3), suggesting that different cancerous stem cells may have different pluripotent potentials. In view of the broad pluripotency in mirPS-Colo cells, we therefore 

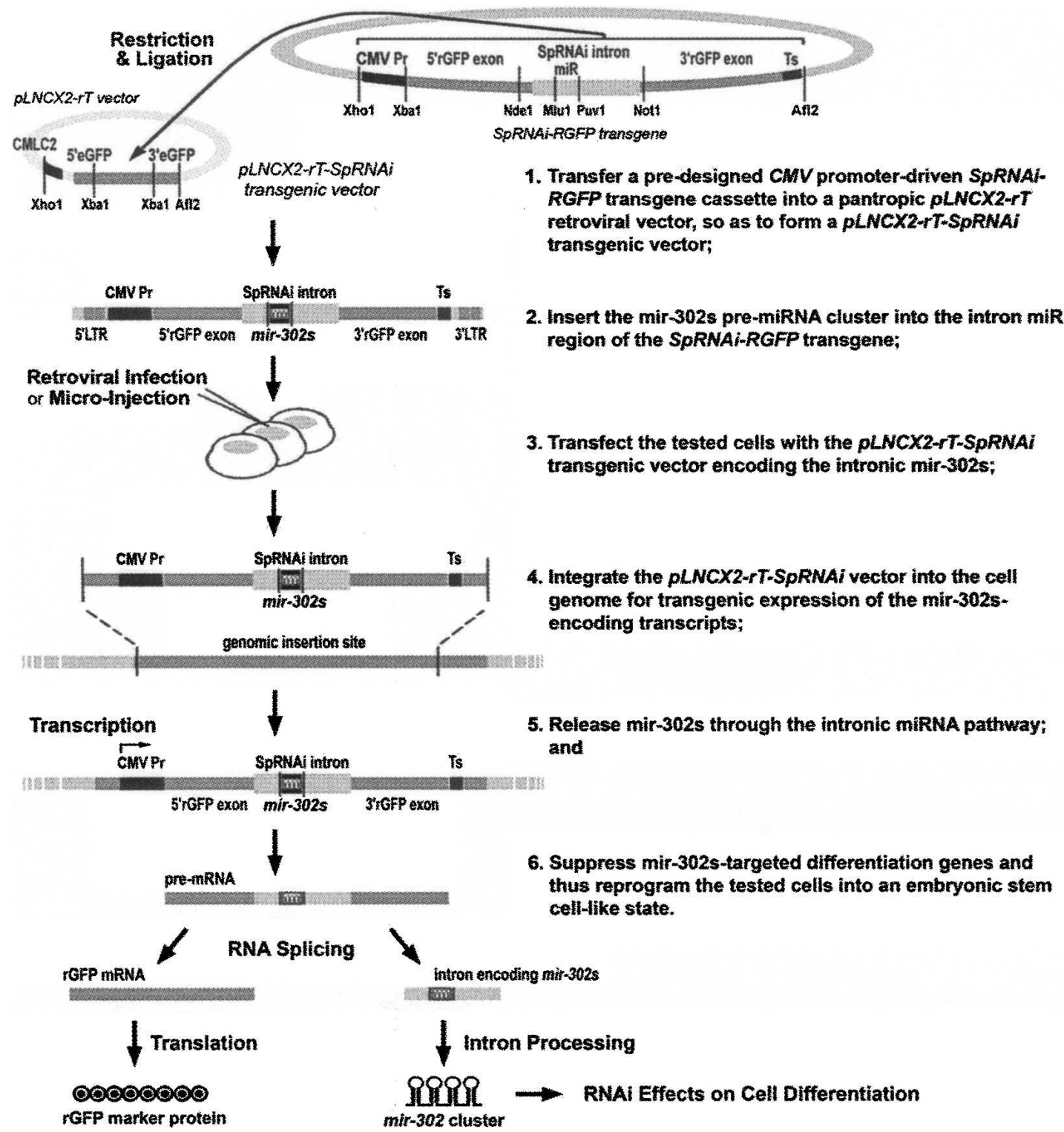

FIGURE 1. Strategy for generating transgenic mir-302s-expressing mirPS cell lines, using retrovirus-based $p L N C X 2-r T-S p R N A i$ vector transfection. A retroviral delivery approach was used to integrate a cytomegalovirus (CMV) promoter-driven SpRNAi-RGFP transgene into the tested cell genomes for steady expression of a manually redesigned mir-302 pre-miRNA cluster (mir-302s). Mir-302s was placed in the intron of the SpRNAi-RGFP transgene and generated as a part of the transgene transcript RNA (pre-mRNA), containing RGFP protein-coding exons and noncoding introns. The introns were spliced out of pre-mRNA and further excised into small miRNA-like mir-302 molecules capable of triggering targeted gene silencing, while the RGFP exons were ligated together to form a mature mRNA for synthesis of a red fluorescent marker protein, RGFP. The presence of RGFP served as an indicator for mir-302s expression and processing.

evaluated the correlation between mir-302 and ES marker expression within the mirPS-Colo cells. As shown in Figure 4A and Supplemental Figure 3, miRNA microarray analyses demonstrated that the expression of each mir-302 member was significantly increased over eightfold in the mirPS-Colo cells. Since the four mir-302 members share very high homology and target almost the same cellular genes, this result indicates that the overall gene silencing effects of mir302s may increase over 30-fold in the mirPS cells. Genomic PCR and fluorescent in situ hybridization assays further revealed that all mirPS cells carried either one or two copies of the mir-302s transgene (Supplemental Fig. 4). Thus, the concentration of mir-302s may affect both pluripotency and survival of the mirPS cells.

\section{Identification of human ES cell markers}

Consistent with the elevation of mir-302 expression, many human ES cell markers were strongly detected in the mirPS cells, as determined by Western blot analyses (Fig. 4B; 


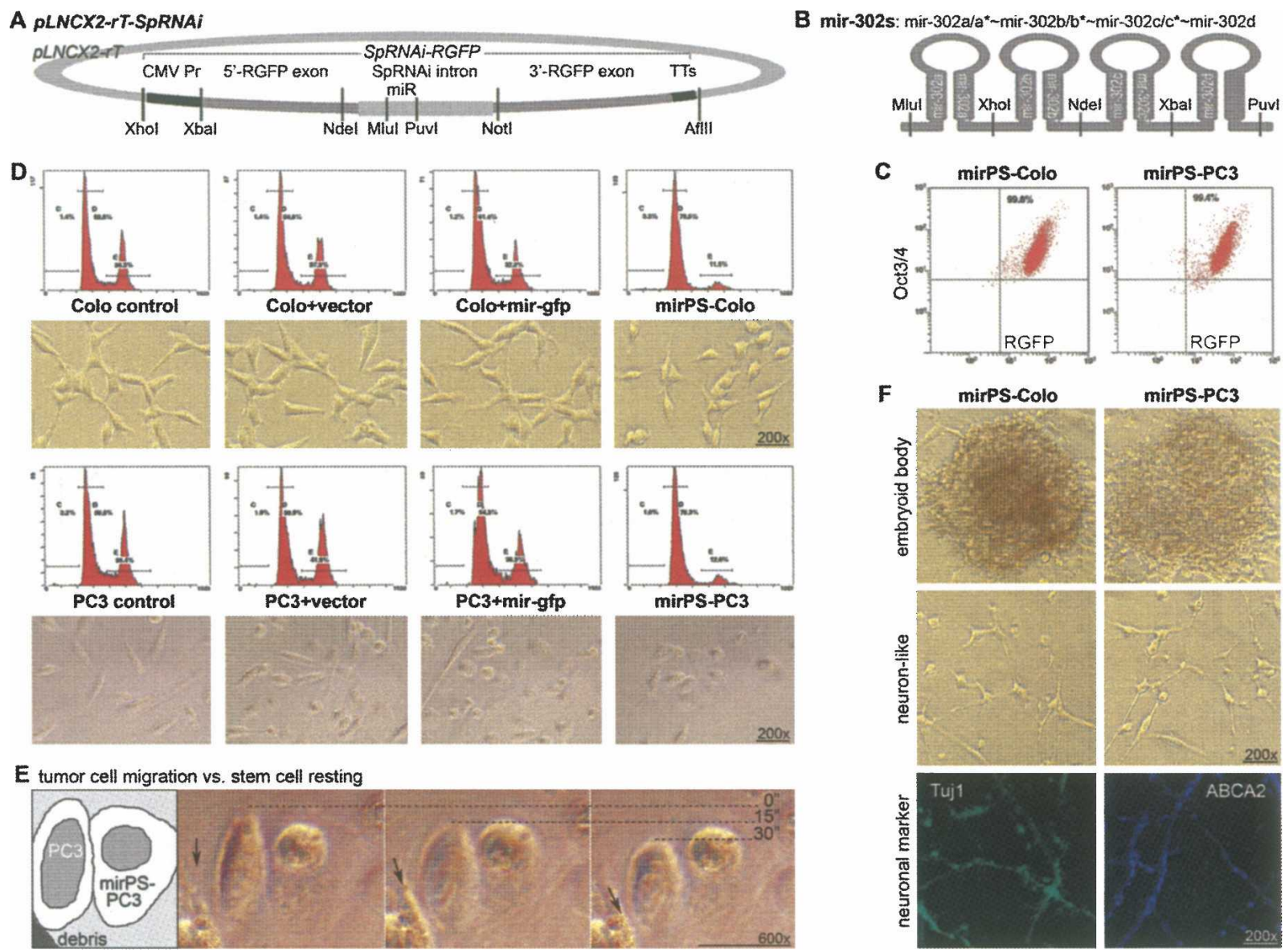

FIGURE 2. Reprogramming of human cancerous Colo and PC3 cells into ES-like mirPS cells with retrovirus-mediated mir-302s transfection. (A) Structure of a mir-302s-expressing SpRNAi-RGFP transgene located in the XhoI/AflII cloning site of a cytomegalovirus (CMV)-promoter-driven pLNCX2 retroviral vector (Clontech), namely, pLNCX2-rT-SpRNAi. (B) Construct of the mir-302 pre-miRNA cluster (mir-302s), which was inserted in the intron region of the SpRNAi-RGFP transgene. (C) Selection of mirPS cells using FACS flow cytometry sorting with RGFP and Oct3/4. (D) Changes of morphologies and cell division rates in mirPS cells. The first (left) and second (right) peaks of the DNA-density flow cytometry charts represented the levels of resting G0/G1 and mitotic M-phase cell populations in the entire tested cell population, respectively. The mir-gfp miRNA shared no homology with human genes. (E) Loss of migration ability in mirPS-PC3 cells compared with its original PC3 cells. $(F)$ Formation of EBs derived from mirPS cells and their differentiation into neuron-like primordial cells with Tuj1 and ABCA2 marker expression.

Supplemental Fig. 5). These ES markers, including Oct3/4, SSEA-3, SSEA-4, Sox2, and Nanog, were barely detected in both the original Colo cancer cells and the cells transfected with an empty $p L N C X 2-r T-S p R N A i$ vector (Colo+vector), a vector expressing mir-gfp miRNA (Colo+mir-gfp), or a vector expressing nonhomologous mir-434-5p pre-miRNA (Colo+mir-434-5p). It has been shown that many central regulators of ES identity, such as Oct3/4, Sox2, and Nanog, are not broadly expressed in high-grade cancers (BenPorath et al. 2008) and the concurrent expression of Oct3/4, SSEA-3, and SSEA-4 is a key determinant of human ES cell identity (Thomson et al. 1998). Therefore, activation of these ES markers in mirPS-Colo cells indicates that mir302s may provide certain "stemness" in order to reprogram the cancerous Colo cells into a human ES-cell-like state. As the TARGETSCAN and PICTAR-VERT programs have predicted that methyl-CpG binding proteins, MECP2 and
MECP1 component p66 (MECP1-p66), are both strong targets of mir-302s, suppression of nuclear DNA methylation seems a very likely mechanism underlying this reprogramming process.

\section{Assessment of reprogramming-related genomic demethylation}

Change of epigenetic modification underlines another unique feature of ES cells, particularly genomic demethylation (Hochedlinger and Jaenisch 2006). In order to reprogram a cell into its ES state, many embryonic genes need to be reactivated by DNA demethylation, such as Oct3/4. To assess this effect in the mirPS cells, we first performed whole genome digestion with HpaII, a restriction enzyme that is sensitive to $\mathrm{CpG}$ methylation and cleaves only an unmethylated CCGG rather than a methylated 


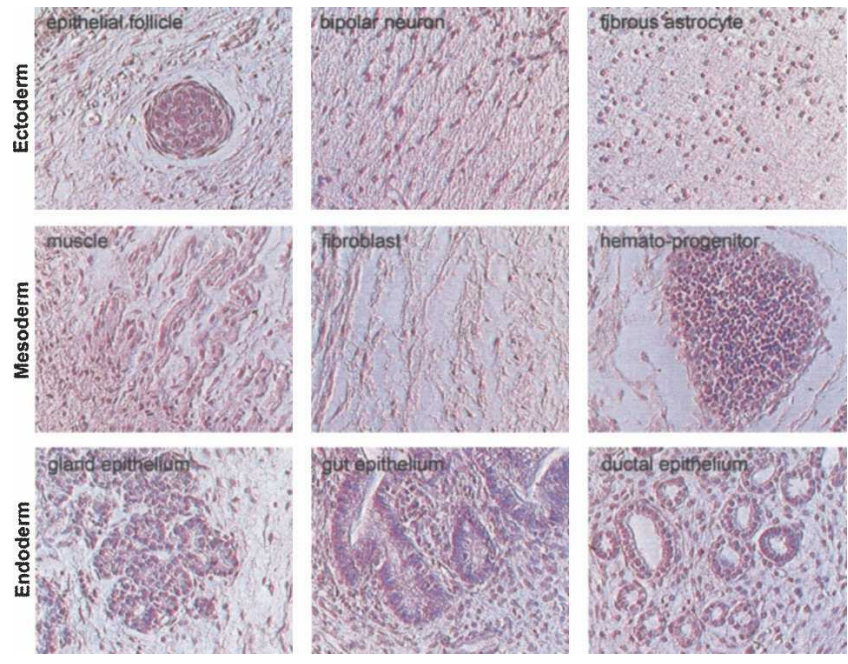

FIGURE 3. Teratoma-like primordial tissues derived from the mirPS EB implants in the uterus or peritoneal cavity of female pseudopregnant immunocompromised SCID-beige mice $(n /$ total $=6 / 6)$. These differentiated tissues included all three embryonic germ layers-ectoderm, mesoderm, and endoderm, as determined by their distinct cell morphologies after hematoxylin and eosin (H \& E) staining. Photographs were taken with the Nikon TE2000 microscopic system at $200 \times$ magnification.

CCGG site. Figure 4C shows that the digested DNA fragments from control Colo cells are over twice as large as those from the mirPS-Colo cells, indicating that the mirPS genome is highly demethylated. Further assessment in the Oct3/4 gene promoter region was performed using bisulfite PCR and genomic DNA sequencing (Takahashi and Yamanaka 2006), which converted all unmethylated cytosines to uracils. Because unmethylated ACGT sites were also changed into AUGT sites by bisulfite, the digestion of mixed ACGT-cutting restriction enzymes failed to cleave this isolated region from the mirPS cells (Fig. 4D). The detailed demethylation maps shown by the bisulfite sequencing further demonstrated that over $90 \%$ methylation sties of the Oct3/4 gene promoter region were lost in the mirPS cells (Fig. 4E), suggesting that an epigenetic reprogramming process did occur to reactivate the Oct3/4 expression. Such epigenetic reprogramming is correlated to mir-302s expression because no DNA demethylation was found in cells transfected with an empty mir-302-free vector compared with control cancer cells.

\section{Microarray analysis of genome-wide gene expression profiles}

Genome-wide gene profiling was required to determine the genetic alterations associated with this mir-302-mediated reprogramming event. Microarray analysis was used to screen changes in genome-wide gene expression patterns in cells before and after the transfection of mir-302s, as well as between the mirPS cells and human ES H1 and H9 cells.
The changes in over 47,000 human gene expression patterns were assessed using Affymetrix gene microarrays (GeneChip U133A\&B and U133 plus 2.0 arrays). We first duplicated the microarray tests using the same mirPS sample and selected 200 most variable genes from one of the tests for further comparison. As shown in Figure 5A, the changes of these selected gene expressions were all less than onefold in the duplicated tests, indicating that the background variation was limited. Based on the scattering patterns of all microarray-identified genes, we then calculated the correlation coefficiency (CC) between the results of two compared transcriptome libraries. A CC rate was given to show the percentage of similarity in the genomewide gene expression patterns with a threshold of only a onefold change. Under such stringent CC rate definition, we found that the gene expression patterns of mirPS-Colo cells were very similar to those of ES H1 (89\%) and H9 (86\%) cells, whereas only a low 53\% CC rate was shown between Colo and mirPS-Colo cells. This strong genetic correlation between human ES and mirPS cells suggests that mir-302s may alter thousands of cellular gene expressions, which are involved in the reprogramming process of a cancer cell into an ES-like mirPS cell. For example, the elevation of many ES gene expressions and shutdown of numerous oncogenic, developmental, and mir-302-targeted cell cyclerelated genes were consistently and concurrently observed in the mirPS and human ES results, as shown in Figure 5, B-E.

In Figure 5E, we noted that cell-cycle checkpoint genes, i.e., CDK2, cyclin D1, and D2, and DNA methylation facilitator, i.e., MECP2 and MECP1-p66, were all confirmed to be strong targets for mir-302s (Supplemental Fig. 5). It is known that cyclin E-dependent CDK2 is required for the entry of S-phase cell cycle and inhibition of CDK2 results in G1-phase checkpoint arrest, whereas cyclin D1 can override G1-phase arrest in response to DNA damage (Sherr and Roberts 2008). Based on this principle, the suppression of both CDK2 and cyclin D1 in mirPS cells revealed a fact that the cell cycle of mir-302-transfected cancer cells could reach a very slow cell division rate, as shown in Figure 2D. The result of such cancer-stem cell cycle transition may provide a significant benefit in cancer therapy. In addition, the suppression of MECP2 and MECP1-p66 activities was consistent with the results of Figure 4, C-E, which indicated the epigenetic reprogramming of malignant cancer cells into benign mirPS cells. It is conceivable that the mirPS cells so obtained from patients may further help to repair the tissue damages of cancers.

\section{In vitro molecular guidance of mirPS cell differentiation}

Pluripotency defines the most important characteristic of an ES cell. Through in vitro manipulations with different factors and/or hormones, human ES cells can differentiate into the three embryonic germ layers (ectoderm, mesoderm, 


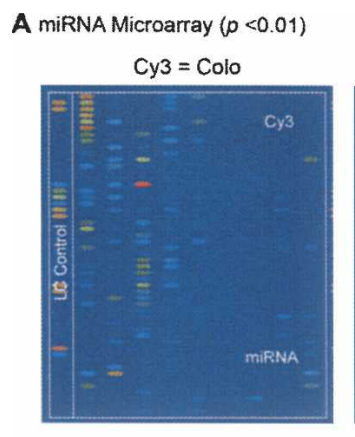

C

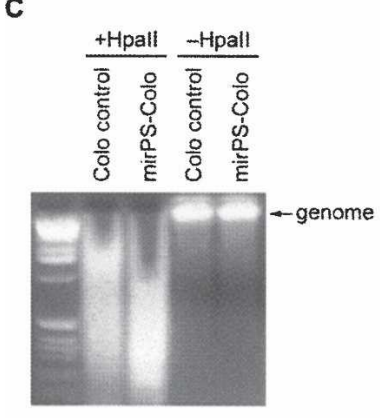

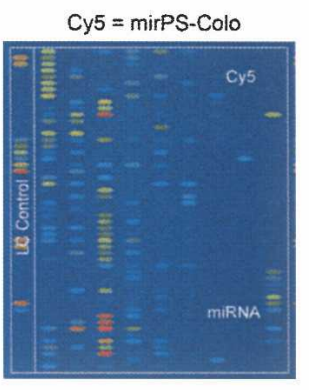

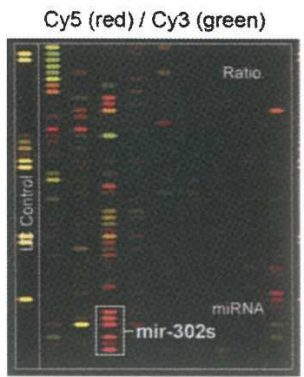

D

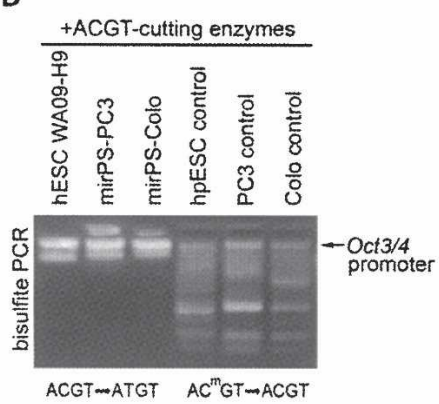

E
B Westem Blotting $(p<0.01)$ 응
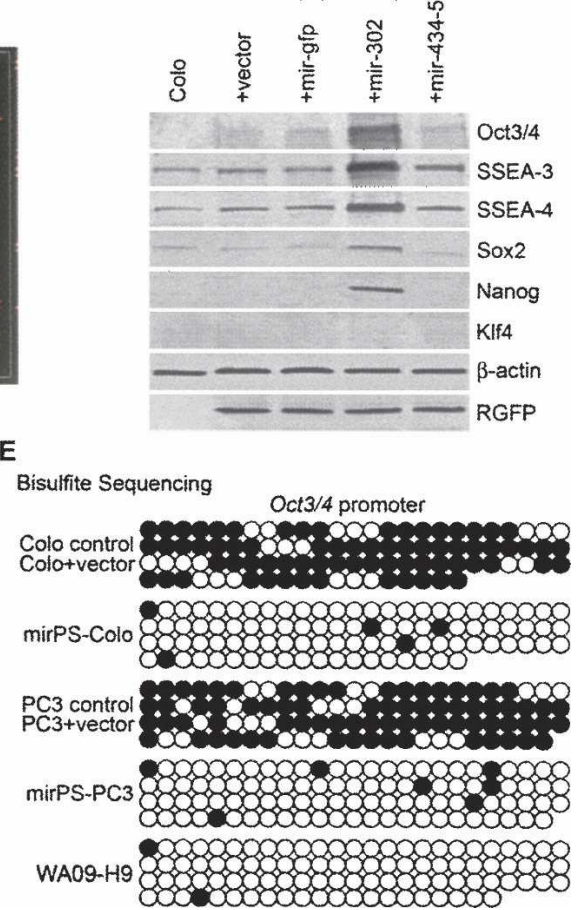

FIGURE 4. Correlation among mir-302 transfection, ES marker expression, and genomic DNA demethylation in mirPS cells. (A) Microarray analyses of miRNA expression, revealing that all mir-302 familial members (mir-302s) were highly expressed in the mirPS-Colo rather than original Colo cells $(\mathrm{n}=3, P<0.01)$. (B) Western blot analyses, showing that mirPS cells expressed high levels of human ES cell markers, including Oct3/4, SSEA-3, SSEA-4, Sox2, and Nanog, but not oncogenic Klf4 $(\mathrm{n}=4, P<0.01)$. (C) HpaII cleavage showing the loss of global CpG methylation at a genome-wide scale in mirPS cells. $(D)$ Bisulfite modification of unmethylated ACGT into AUGT sites in the 9400-bp regulatory region of the Oct3/4 promoter, showing an increase of unmethylated ACTG (AUCT) sites in mirPS cells. (E) Bisulfite DNA sequencing, showing the detailed methylation maps flanking the transcription initiation site of the Oct3/4 promoter. Black and white circles indicate the methylated and unmethylated cytosine sites, respectively.

and definitive endoderm) — the founders of all adult tissues. In the absence of any treatment, xenograft implantation of the mirPS-Colo-derived EBs into the uterus or peritoneal cavity of female pseudopregnant, immunocompromised SCID-beige mice formed teratoma-like primordial tissue cysts (Fig. 3). The growth of these teratoma-like structures was terminated $\sim 2.5$ wk post-implantation. It seems that there is a self-regulation mechanism limiting the random growth of these mirPS EB cells in vivo. However, using in vitro treatments of various growth factors and/or hormones, we could direct the mirPS-Colo cells to differentiate into several tissue cell types ex vivo, including fibroblasts (Fig. 6A-E), chondrocytes (Fig. 6F-J), and spermatogonialike (Fig. 6K-O) primordial cells. The protocols for these in vitro conditions and xenotransplantation methods are provided in the Materials and Methods. Markers for the special tissue lineages were also identified with immunohistochemical detection, showing germ line-specific Dazla and EE2, fibroblast-specific atlastin1 and type I pro-collagen (COL1A1), and chondrocyte-specific tropoelastin and type II pro-collagen (COL2A1), respectively. These findings confirmed the pluripotency of the mirPS cells. It is conceivable that many more tissue cell types may be induced from these mirPS cells, using different molecular interventions.

\section{DISCUSSION}

Ever since the first isolation of human ES cell lines from human blastocysts (Thomson et al. 1998), there were concerns about destruction of human embryos, contamination of feeder cell antigens, and formation of teratomas. Recent reports on induced pluripotent stem (iPS) cells have opened up a new avenue for generating ES-like pluripotent cells directly from adult body cells, bypassing the use of human embryos as starting materials (Takahashi and Yamanaka 2006; Takahashi et al. 2007). Using retroviral delivery of four transcription factor genes (i.e., either Oct4Sox2-c-Myc-Klf4 or Oct4-Sox2-Nanog-Lin28) into mouse embryonic fibroblasts, the iPS cells so obtained were similar in many genetic and behavioral properties to mouse ES cells (Okita et al. 2007; Wernig et al. 2007). Additional iPS cell lines have continued to be developed from human embryonic fibroblasts and primary dermal fibroblast cultures using a similar approach (Yu et al. 2007; Park et al. 2008). Yet, there are two problems emerging from the 
A

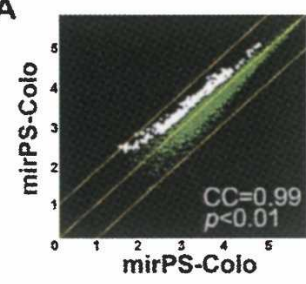

B
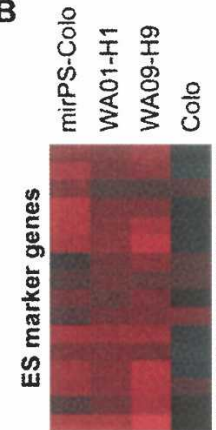

D

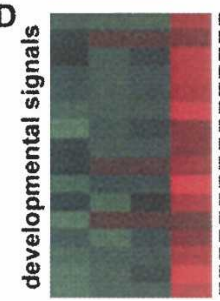

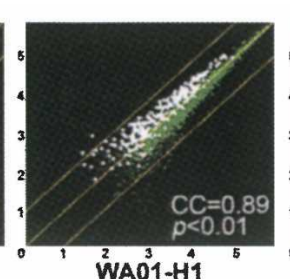

WAO1-H1

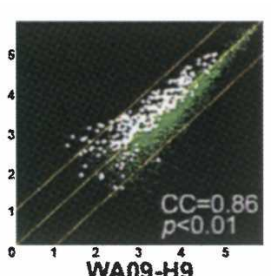

C
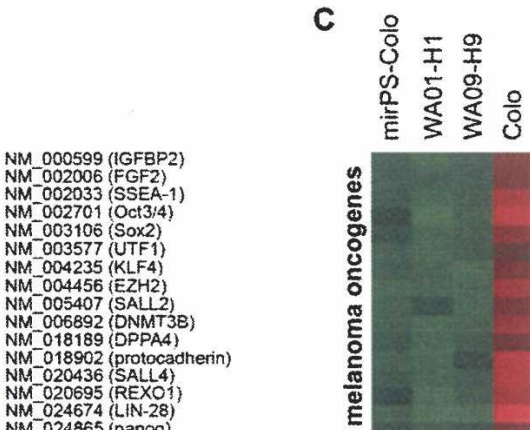
NM- 024865 (nanog)

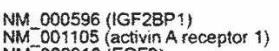
NM-001105 (activin A $\mathrm{NM}-002010$ (FGF9)
$\mathrm{NM}-002193$ (inhibin 3 (3b) NM 002193 (inhibin 3 (3D)
NM 002397 (MEF 2 )
NM 002752 (MAPKS) NM-002752 (MAPK9) NM-005117 (FGF19) NM-014368 (LIM hormeobox 8) NM-0 016269 (LEF1)
NM-018416 (FOXJ2) NM-020997 (LEFTY1) NM- 022123 (NPAS3) NM_15716 (MINK)
NM_202394 (E2F7)

level

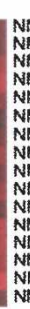

E

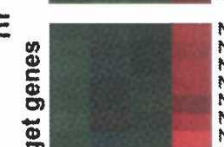
NM-022763 (FAD104
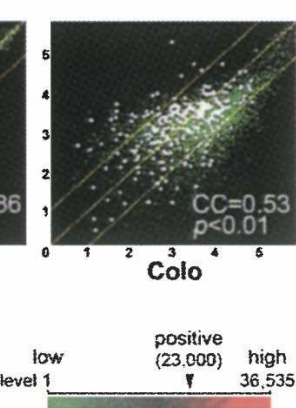

NM 001754 (AAKL1) MM-002655 (PLAG1) NM- 003189 (TAL 1) NM- 005235 ( $(\mathrm{B}$ - Esb homolong 4$)$ NM- $005378\left(\mathrm{v}-\mathrm{Mrhy}_{\mathrm{C}} \mathrm{c}\right)$ NM_006292 (TSG101) NM-012197 (Rab-GAP1) NA: 015094 (HIC2) NM-015313 (RholRac GEF) NM-016544 (Rap GEF) NM-018098 (ECT2) NM- 020310 (MNT) NM
NM 020673 (Rab member of Ras oncogene)

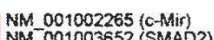

NiM 001002265 (C-Mir)
0003652 (SMAD2) M 001759 (cyclin D2) MM 001798 (CDK2) NM-004992 (MECP2) NA- 006037 (HDAC4) NM 006766 (MYST3) NM-012199 (EIF2C1) NM-012257( $\mathrm{HBP}$ 1) MM- 015215 (CAMTA NM 020699 (P66R, MECP i component) NM-021644 (nudear RNP H3) NM-053056 (yclin D1)

FIGURE 5. Genome-wide gene expression analyses among Colo, mirPS-Colo, and human ES WA01-H1 (H1) and WA09-H9 (H9) cells. (A) Comparison of altered gene expression patterns using Human genome GeneChip U133A\&B and plus 2.0 arrays (Affymetrix), showing high similarity between mirPS-Colo and $\mathrm{H1}(89 \%)$ as well as $\mathrm{H9}$ (86\%), but not original Colo $(53 \%)$ cells. White dots referred the highly variable genes compared with the stably expressed genes (green dots). (B-E) Functional clustering of microarray-identified differentially expressed genes, demonstrating that a significant increase of ES cell markers $(B)$ and a marked decrease of melanoma oncogenes $(C)$, developmental signals $(D)$, and mir-302stargeted cell proliferation and DNA methylation genes $(E)$ were detected in mirPS cells, which highly resembled those in $\mathrm{H} 1$ and $\mathrm{H} 9$ cells $(\mathrm{n}=4, \mathrm{P}<0.01)$. Any signal showing above the level 23,000 of a total 36,535 (in red) was considered to be a positive call in the gene expression list. All differential genes shown in $C-E$ were the target genes of mir-302s predicted by the TARGETSCAN and PICTAR-VERT programs.

process of iPS cell generation; one is the use of retroviral transgenes and the other the use of oncogenes (e.g., $c-M y c$ and Klf4). Retroviral transfection is the only effective means to simultaneously and transgenically deliver the four fulllength genes into a targeted somatic cell, whereas the random insertion of retroviral vectors into the transfected cell genome may also affect other nontargeted genes and produce unexpected results. This is problematic because simultaneous delivery of four large transgenes into one single cell is difficult to control, particularly when one or more of the transgenes are oncogenes.

Unlike the previous iPS cell technology, each member of the mir-302 family is able to simultaneously regulate over 445 cellular genes and they all share almost the same target genes. Many of the mir-302 targeted genes are active developmental signals involved in initiation or facilitation of lineage-specific cell differentiation during early embryonic development. By suppressing these developmental genes, mir-302s may reprogram cells into a more ES-like state. Alternatively, mir-302s may also attenuate the expression of their predicted target transcription factors, such as SP3, HMG-box, forkhead-box, and LIM-homeobox gene families, to provide the cell reprogramming effect. Thus, the function of mir$302 \mathrm{~s}$ is more likely to attenuate the global production of developmental signals and/or transcription factors rather than to directly create transcriptional stimulation on certain embryonic signaling pathways. By inhibiting the cellular genes essential for embryonic development and cell differentiation, mir-302s is able not only to reprogram differentiated cancer cells into ES-like pluripotent stem cells but also to maintain their pluripotency and renewal under a feeder-free culture condition. Nevertheless, mir-302s may not be the only miRNA family involved in this mechanism because their target genes are also redundantly silenced by the group of mir-93, mir-367, mir-371, mir-372, mir-373, and mir-520 in human ES cells, as predicted by the TARGETSCAN program. Learning why these target genes must be simultaneously silenced during the reprogramming process of cancer-ES cell transformation may shed light on the mechanism underlying this miRNA-mediated effect on ES cell maintenance and renewal.

Utilization of intronic mir-302 transfection provides a safe and powerful new tool for generation of human ES-like pluripotent stem cells, particularly derived from cancerous and primarily cultured somatic cells. Because the intronic miRNA pathway is tightly regulated by multiple intracellular surveillance systems, such as mRNA transcription, RNA splicing, exosomal digestion, and nonsense-mediated decay (NMD) mechanisms, it is considered to be much more effective, specific, and safe than the siRNA/shRNA pathway (Lin et al. 2008). Advantageously, there are five breakthroughs in this method of mir-302-mediated mirPS cell generation. First, the transfection of a single mir-302s-expressing transgene offers a very simple, efficient, and safe method for generating homogeneous ES-like pluripotent cells, preventing the tedious retroviral insertion of all four large transcription factor genes into one single target cell as demonstrated 

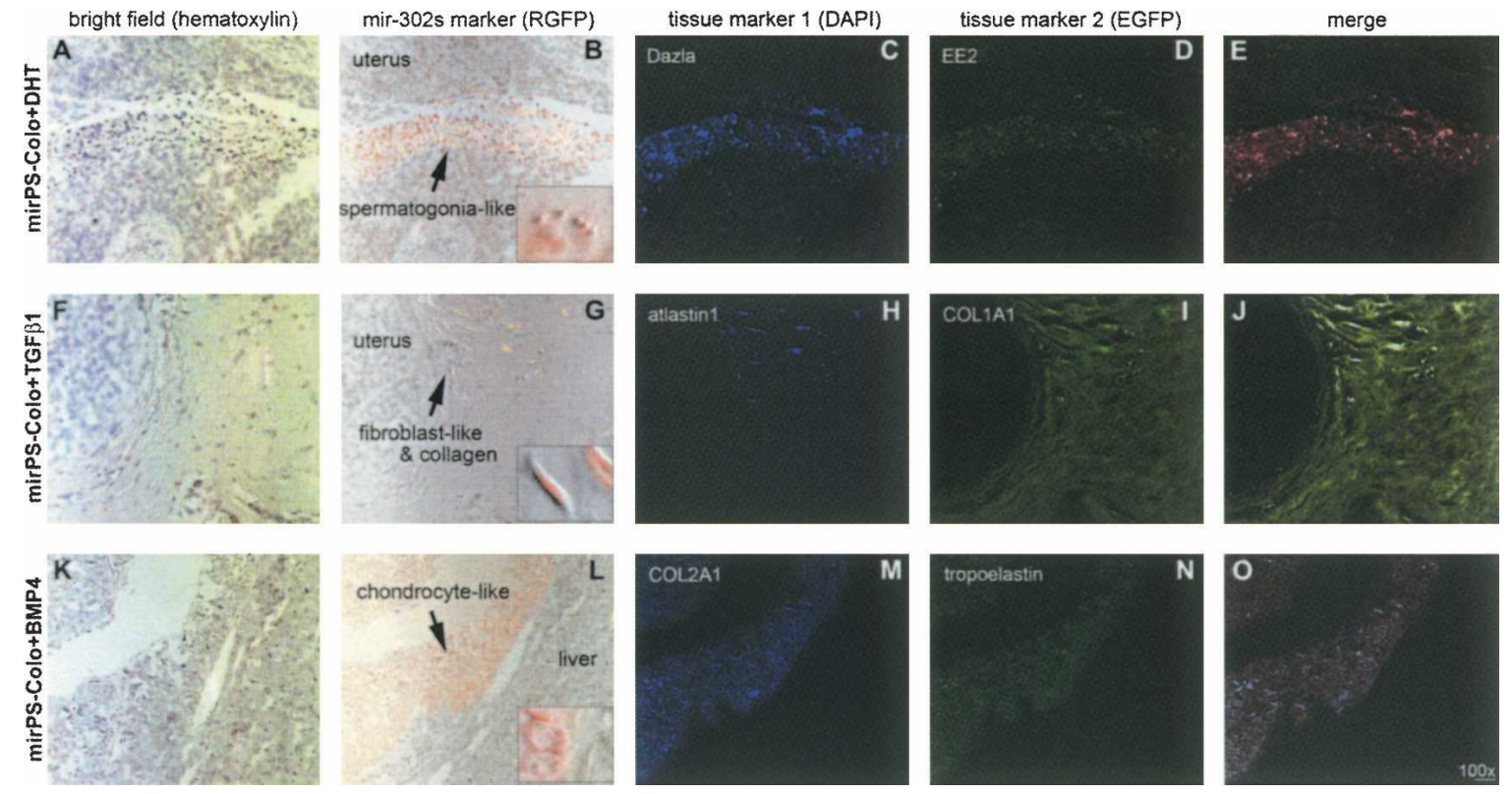

FIGURE 6. Pluripotency of mirPS cells. Treatments of DHT, TGF- $\beta 1$ and BMP4, respectively from top to bottom, induced the mirPS-Colo cell differentiation into spermatogonia-like $(A-E)$, fibroblast-like $(F-J)$, and chondrocyte-like $(K-O)$ primordial cells, in immunocompromised mice ex vivo. The use of immunocompromised nude mice was to provide an in vivo environment mimicking transplantation therapy. Microscopic photographs shown from left to right indicated hematoxylin staining with differential interference contrast $(A, F, K)$, bright field labeled with transgenic mir-302 marker RGFP (red) ( $B, G, L)$, immunostaining of the first tissue marker labeled with 4,6-diamidino-2-phenylindole (blue DAPI) $(C, H, M)$, immunostaining of the second tissue marker labeled with fluorescein (green EGFP) $(D, I, N)$, and merge of all three fluorescent markers $(E, J, O)$. Small windows in the RGFP-bright fields showed the morphologies of differentiated mirPS cells at high magnification $(600 \times)$.

in the previous iPS methods. Second, because the size of the mir-302s transgene is merely $\sim 1 \mathrm{~kb}$, the transfection efficiency is extremely high $(\sim 100 \%)$ and the positive mirPS cells can be easily selected by one pass through FACS flow cytometry, which is a very time-saving process. Third, the transfection and cultivation of mirPS cells can be performed under a feeder-free condition without the risk of feeder antigen contamination. Fourth, no oncogene is used for mirPS cell generation. Last, we may use electroporation in place of retroviral transfection to deliver the single mir302 transgene, preventing the risk of random viral insertion into the host cell genome. Given that these advantages have solved most of the problems found in current stem cell research, learning how to use these mirPS cells for exploiting their potentials in transplantation and cell therapies will be a forthcoming challenge in the near future.

\section{MATERIALS AND METHODS}

\section{Cell culture and treatments}

Human cancer cell lines, Colo, and PC3 cells were purchased from the American Type Culture Collection (ATCC) and cultivated in RPMI 1640 medium supplemented with $10 \%$ fetal bovine serum (FBS), $4 \mathrm{mM} \mathrm{L}$-glutamine, $1 \mathrm{mM}$ sodium pyruvate, and $100 \mu \mathrm{g} /$ $\mathrm{mL}$ gentamycin (Sigma Chemical), at $37^{\circ} \mathrm{C}$ under $5 \% \mathrm{CO}_{2}$. Cultures were passaged at $\sim 80 \%$ confluency by exposing cells to trypsin-EDTA solution for $1 \mathrm{~min}$ and rinsing once with RPMI, and the detached cells were replated at 1:10 dilution in fresh growth medium. After mir-302 transfection, mirPS-Colo and mirPS-PC3 cells were grown on polyornithine/laminin-coated dishes, respectively, in a freshly made mirPS cell culture medium, containing either phenol red-free DMEM/F12 (1:1; high glucose) or RPMI 1640/B27 medium (Invitrogen) supplemented with 10\% charcoal-stripped FBS, $4 \mathrm{mM}$ L-glutamine, $1 \mathrm{mM}$ sodium pyruvate, $5 \mathrm{ng} / \mathrm{mL}$ activin, $5 \mathrm{ng} / \mathrm{mL}$ noggin, $3 \mathrm{ng} / \mathrm{mL}$ bFGF (BD Biosciences), and an equal mixture of $0.5 \mu \mathrm{M} \mathrm{Y}-27632$ and 0.5 $\mu \mathrm{M}$ GSK-3 inhibitor XV (EMD Biosciences), at $37^{\circ} \mathrm{C}$ under $5 \%$ $\mathrm{CO}_{2}$. The mirPS cells were passaged at $85 \% \sim 90 \%$ confluency by exposing cells to trypsin-EDTA/collagenase IV $(1 \mathrm{mg} / \mathrm{mL})$ solution for $1 \mathrm{~min}$ and rinsing once with DMEM/F12 medium. Detached cells were replated at 1:3 dilution in fresh growth medium supplemented with $30 \%(\mathrm{v} / \mathrm{v})$ conditioned medium that had exposed to the cells for $24 \mathrm{~h}$ before passaging.

\section{Construction of the SpRNAi-RGFP transgene encoding an intronic mir-302 pre-miRNA cluster insert}

The SpRNAi-RGFP transgene was generated as reported in the Supplemental Materials and Methods (Lin and Ying 2006; Lin et al. 2006). The intronic mir-302 pre-miRNA cluster consists of four parts: mir-302a, mir-302b, mir-302c, and mir-302d premiRNAs. Synthetic oligonucleotides were as follows:

mir-302a-sense, 5'-GTCCGATCGTCCCACCACTTAAACGTGG ATGTACTTGCTTTGAAACTAAAGAAGTAAGTG CTTCCAT GTTTTGGTGATGGATCTCGAGCTC-3'; 
mir-302a-anti-sense, 5' -GAGCTCGAGATCCATCACCAAAACAT GGAAGCACTTACTTCTTTAGTTTCAAAGCAAGTACATCC ACGTTTAAGTGGTGGGACGATCGGAC-3';

mir-302b-sense, 5' -ATCTCGAGCTCGCTCCCTTCAACTTTAAC ATGGAAGTGCTTTCTGTGACTTTGAAAGTAAGTGCTTCC ATGTTTTAGTAGGAGTCGCTAGCGCTA-3';

mir-302b-anti-sense, 5' -TAGCGCTAGCGACTCCTACTAAAACA TGGAAGCACTTACTTTCAAAGTCACAGAAAGCACTTCCA TGTTAAAGTTGAAGGGAGCGAGCTCGAGAT-3';

mir-302c-sense, 5'-CGCTAGCGCTACCTTTGCTTTAACATGGA

GGTACCTGCTGTGTGAAACAGAAGTAAGTCG TTCATGTT

TCAGTGGAGGCGTCTAGACAT-3';

mir-302c-anti-sense, 5'-ATGTCTAGACGCCTCCACTGAAACA

TGAACGACTTACTTCTGTTTCACACAGCAGGTACCTCCA

TGTTAAAGCAAAGGTAGCGCTAGCG-3';

mir-302d-sense, 5'-CGTCTAGACATAACACTCAAACATGGAA

GCACTTAGCTAAGCCAGGCTAAGTGCTTCCATGTTTGAG

TGTTCGACGCGTCAT-3'; and

mir-302d-anti-sense, 5'-ATGACGCGTCGAACACTCAAACATG

GAAGCACTTAGCCTGGCTTAGCTAAGTGCTTCCATGTTT GAGTGTTATGTCTAGACG-3'.

(Sigma-Genosys). We first hybridized mir-302a-sense to mir-302aanti-sense, mir-302b-sense to mir-302b-anti-sense, mir-302c-sense to mir-302c-anti-sense, and mir-302d-sense to mir-302d-antisense, respectively, for $2 \mathrm{~min}$ at $94^{\circ} \mathrm{C}$, for $10 \mathrm{~min}$ at $70^{\circ} \mathrm{C}$, and then at $4^{\circ} \mathrm{C}$ in $1 \times$ PCR buffer. Then, the same amount of each hybrid of mir-302a, mir-302b, mir-302c, and mir-302d were digested with PvuI/XhoI, XhoI/NheI, NheI/XbaI, and XbaI/MluI restriction enzymes, respectively, at $37^{\circ} \mathrm{C}$ for $4 \mathrm{~h}$. All of the digested hybrids were collected together with a gel extraction filter in $35 \mu \mathrm{L}$ of autoclaved $\mathrm{ddH}_{2} \mathrm{O}$ (Qiagen). Immediately after that, the mir-302 pre-miRNA cluster was formed by adding T4 DNA ligase $(20 \mathrm{U})$ and buffer into the hybrid mixture and incubating the reaction at $12^{\circ} \mathrm{C}$ for $12 \mathrm{~h}$. For intronic insertion, we mixed an equal amount $(1: 1)$ of the mir-302 pre-miRNA cluster and the SpRNAi-RGFP transgene and then digested the mixture with PvuI and MluI restriction enzymes for $4 \mathrm{~h}$ at $37^{\circ} \mathrm{C}$. The digested mixture was collected with a microcon-30 filter and ligated together with $\mathrm{T}_{4}$ DNA ligase $(20 \mathrm{U})$ for $12 \mathrm{~h}$ at $12^{\circ} \mathrm{C}$. This formed the SpRNAi-RGFP transgene with the intronic mir-302 premiRNA cluster insert.

\section{Incorporation of the SpRNAi-RGFP transgene into a pLNCX2-rT-SpRNAi retroviral vector}

We modified a $V S V$-G-positive pantropic retroviral vector, i.e., pLNCX2-rT, to transgenically deliver the mir-302-encoded $S p R N A i-R G F P$ transgene (Lin et al. 2006). The $p L N C X 2-r T$ vector was derived from a modified pseudotype Moloney Murine Leukemia virus, $p L N C X 2$ (Clontech). As shown in Figure 1, we first incorporated the SpRNAi-RGFP transgene into the XhoI/AflII restriction site of the $p L N C X 2-r T$ vector and then inserted the mir-302 pre-miRNA cluster construct into the intronic insertion site (PvuI/MluI restriction site) of the SpRNAi-RGFP transgene, so as to form a retroviral $p L N C X 2-r T-S p R N A i$ transgene vector capable of transgenically expressing mir-302s. In experiments, we mixed an equal amount (1:1) of the SpRNAi-RGFP transgene and the $p L N C X 2-r T$ retroviral vector and then digested the mixture with XhoI and AflII restriction enzymes for $4 \mathrm{~h}$ at $37^{\circ} \mathrm{C}$. The digested mixture was collected with a microcon-30 filter and ligated together with $\mathrm{T}_{4}$ DNA ligase (20 U, Roche Biochemicals) for $12 \mathrm{~h}$ at $12^{\circ} \mathrm{C}$. Following the same protocol except using PvuI/ MluI digestion, we incorporated the mir-302 pre-miRNA cluster into the PvuI/MluI restriction site of the $p L N C X 2-r T-S p R N A i$ vector. The pre-miRNA-inserted $p L N C X 2-r T$-SpRNA $i$ vector was propagated in Escherichia coli DH5 $\alpha$ LB cultures containing 100 $\mu \mathrm{g} / \mathrm{mL}$ ampicillin (Sigma) and purified with a QIAprep spin miniprep kit (Qiagen), following the manufacturer's suggestion. For viral production, the $p L N C X 2-r T-S p R N A i$ vector was cotransfected with an equal amount of $p V S V-G$ vector into GP2-293 packaging cells (Clontech) to produce infectious, but not replicable, pantropic retroviruses. GP2-293 cells were grown in phenol red-free DMEM medium supplemented with 10\% FBS, $4 \mathrm{mM}$ L-glutamine, and $1 \mathrm{mM}$ sodium pyruvate. The co-transfection was carried out with a FuGene 6 reagent (Roche), following the manufacturer's suggestion.

\section{MirPS cell generation using the pLNCX2-rT-SpRNAi transgene vector}

High titer viruses were released in the DMEM medium of the GP2-293 cell cultures $\sim 36-48 \mathrm{~h}$ after the cotransfection of $p V S V$ $G$ and $p L N C X 2-r T$-SpRNA $i$ vectors. The viral titer was measured to be over multiplicity of infection (MOI) 30 before cell transfection, following the protocol of a retro-X qRT-PCR titration kit (Clontech). Then, we transferred the high titer virus medium to the Colo or PC3 cell cultures and incubated the cells for $12 \mathrm{~h}$ at $37^{\circ} \mathrm{C}$ under $5 \% \mathrm{CO}_{2}$. After that, fresh mirPS cell culture medium was added in place of the virus medium and replaced every $3 \mathrm{~d}$. Positively transfected cells were isolated and collected $24 \mathrm{~h}$ postinfection, using FACS flow cytometry sorting with a monoclonal antibody against the mir-302 expression maker RGFP (Clontech). These isolated cells were grown in the mirPS cell culture medium as aforementioned.

\section{In vitro molecular guidance of the mirPS cell differentiation}

In absence of any treatment except the feeder-free mirPS culture medium, xenograft implantation of the mirPS EB into the uterus or peritoneal cavity, but not other tissues, of a 6-wk-old female pseudopregnant, immunocompromised SCID-beige mouse could form a cyst full of teratoma-like primordial tissues. The use of immunocompromised nude mice was to provide an in vivo environment mimicking transplantation therapy. The pseudopregnant mice were made by intraperitoneally injection of 1 IU human menopausal gonadotrophin (HMG) for $2 \mathrm{~d}$ and then human chorionic gonadotrophin (hCG) for one more day. For in vitro molecular guidance into spermatogonia lineage, mirPS cells were maintained on polyornithine/laminin-coated dishes in DMEM/F12 (1:1; high glucose) medium supplemented with charcoal-stripped 10\% FBS, $4 \mathrm{mM}$ L-glutamine, $1 \mathrm{mM}$ sodium pyruvate, $5 \mathrm{ng} / \mathrm{mL}$ activin, and $50 \mathrm{ng} / \mathrm{mL}$ dihydrotestosterone (DHT) for $12 \mathrm{~h}$ at $37^{\circ} \mathrm{C}$ under $5 \% \mathrm{CO}_{2}$. Then the cells were trypsinized, washed with $1 \times$ PBS, and collected in four aliquots of chilled Matrigel $(100 \mu \mathrm{L}$ each) and one aliquots of $100 \mu \mathrm{L} 1 \times$ PBS. Immediately after that, we transplanted the cells into the hind limb muscle, peritoneum, uterus, subcutaneous neck skin (with Matrigel), and tail vein (with PBS) of 6-wk-old athymic immunocompromised SCID-beige nude mice. The mice were 
anesthetized with diethyl ether during experimental processing. One week later, spermatogonia-like cells were found only in the uterus area. For fibroblast differentiation, we followed the same procedure as shown above, except using regular phenol red-free DMEM medium supplemented with 10\% FBS, $4 \mathrm{mM}$ L-glutamine, $1 \mathrm{mM}$ sodium pyruvate, $5 \mathrm{ng} / \mathrm{mL}$ noggin, and $100 \mathrm{ng} / \mathrm{mL}$ transforming growth factor- $\beta 1$ (TGF- $\beta 1$ ) for $6 \mathrm{~h}$ before xenotransplantation. Fibroblast-like cells were found in the uterus $1 \mathrm{wk}$ later. For chondrocyte differentiation, we performed the same procedure as before but using regular RPMI 1640 medium supplemented with $10 \%$ FBS, $4 \mathrm{mM}$ L-glutamine, $1 \mathrm{mM}$ sodium pyruvate, and $100 \mathrm{ng} / \mathrm{mL}$ bone morphogenetic protein 4 (BMP4) for $6 \mathrm{~h}$. Chondrocyte-like cells were found only in the liver area.

\section{SUPPLEMENTAL DATA}

Supplemental material can be found at http://www.rnajournal.org.

Received May 1, 2008; accepted July 15, 2008.

\section{REFERENCES}

Bartel, D.P. 2004. MicroRNAs: Genomics, biogenesis, mechanism, and function. Cell 116: 281-297.

Ben-Porath, I., Thomson, M.W., Carey, V.J., Ge, R., Bell, G.W., Regev, A., and Weinberg, R.A. 2008. An embryonic stem cell-like gene expression signature in poorly differentiated aggressive human tumors. Nat. Genet. 40: 499-507.

Danin-Kreiselman, M., Lee, C.Y., and Chanfreau, G. 2003. RNAse IIImediated degradation of unspliced pre-mRNAs and lariat introns. Mol. Cell 11: 1279-1289.

Hochedlinger, K. and Jaenisch, R. 2006. Nuclear reprogramming and pluripotency. Nature 441: 1061-1067.

Lin, S.L. and Ying, S.Y. 2006. Gene silencing in vitro and in vivo using intronic microRNAs. In MicroRNA protocols (ed. S.-Y. Ying), pp. 295-312. Humana Press, Totowa, NJ.

Lin, S.L., Chang, D., Wu, D.Y., and Ying, S.Y. 2003. A novel RNA splicing-mediated gene silencing mechanism potential for genome evolution. Biochem. Biophys. Res. Commun. 310: 754-760.

Lin, S.L., Chang, S.J.E., and Ying, S.Y. 2006. Transgene-like animal model using intronic microRNAs. Methods Mol. Biol. 342: 321-334.
Lin, S.L., Kim, H., and Ying, S.Y. 2008. Intron-mediated RNA interference and microRNA (miRNA). Front. Biosci. 13: 22162230.

Okita, K., Ichisaka, T., and Yamanaka, S. 2007. Generation of germline-competent induced pluripotent stem cells. Nature 448: 313-317.

Park, I.H., Zhao, R., West, J.A., Yabuuchi, A., Huo, H., Ince, T.A., Lerou, P.H., Lensch, M.W., and Daley, G.Q. 2008. Reprogramming of human somatic cells to pluripotency with defined factors. Nature 451: 141-146.

Reya, T., Morrison, S.J., Clarke, M.F., and Weissman, I.L. 2001. Stem cells, cancer, and cancer stem cells. Nature 414: 105-111.

Rodriguez, A., Griffiths-Jones, S., Ashurst, J.L., and Bradley, A. 2004. Identification of mammalian microRNA host genes and transcription units. Genome Res. 14: 1902-1910.

Ruby, J.G., Jan, C.H., and Bartel, D.P. 2007. Intronic microRNA precursors that bypass Drosha processing. Nature 448: 83-86.

Sherr, C.J. and Roberts, J.M. 2008. Living with or without cyclins and cyclin-dependent kinases. Genes \& Dev. 18: 2699-2711.

Suh, M.R., Lee, Y., Kim, J.Y., Kim, S.K., Moon, S.H., Lee, J.Y., Cha, K.Y., Chung, H.M., Yoon, H.S., Moon, S.Y., et al. 2004. Human embryonic stem cells express a unique set of microRNAs. Dev. Biol. 270: 488-498.

Takahashi, K. and Yamanaka, S. 2006. Induction of pluripotent stem cells from mouse embryonic and adult fibroblast cultures by defined factors. Cell 126: 663-676.

Takahashi, K., Tanabe, K., Ohnuki, M., Narita, M., Ichisaka, T., Tomoda, K., and Yamanaka, S. 2007. Induction of pluripotent stem cells from adult human fibroblasts by defined factors. Cell 131: 861-872.

Thomson, J.A., Itskovitz-Eldor, J., Shapiro, S.S., Waknitz, M.A., Swiergiel, J.J., Marshall, V.S., and Jones, J.M. 1998. Embryonic stem cell lines derived from human blastocysts. Science 282: 11451147.

Wernig, M., Meissner, A., Foreman, R., Brambrink, T., Ku, M., Hochedlinger, K., Bernstein, B.E., and Jaenisch, R. 2007. In vitro reprogramming of fibroblasts into a pluripotent ES-cell-like state. Nature 448: $318-324$.

Xia, X.G., Zhou, H., Samper, E., Melov, S., and Xu, Z. 2006. Pol IIexpressed shRNA knocks down Sod2 gene expression and causes phenotypes of the gene knockout in mice. PLoS Genet. 2: e10.

Yu, J., Vodyanik, M.A., Smuga-Otto, K., Antosiewicz-Bourget, J., Frane, J.L., Tian, S., Nie, J., Jonsdottir, G.A., Ruotti, V., Stewart, R., et al. 2007. Induced pluripotent stem cell lines derived from human somatic cells. Science 318: 1917-1920. 

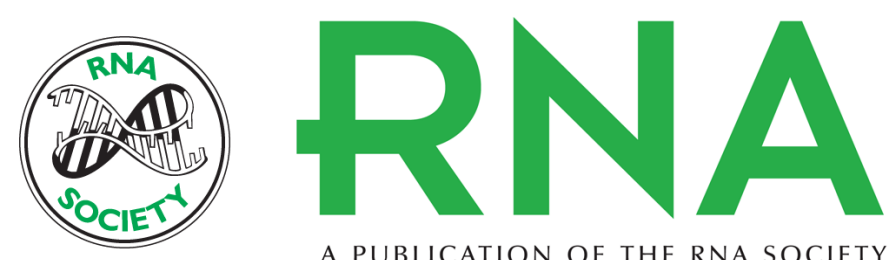

A PUBLICATION OF THE RNA SOCIETY

\section{Mir-302 reprograms human skin cancer cells into a pluripotent ES-cell-like state}

Shi-Lung Lin, Donald C. Chang, Samantha Chang-Lin, et al.

RNA 2008 14: 2115-2124 originally published online August 28, 2008

Access the most recent version at doi:10.1261/rna.1162708

\section{Supplemental http://rnajournal.cshlp.org/content/suppl/2008/08/29/rna.1162708.DC1 \\ Material http://rnajournal.cshlp.org/content/suppl/2008/12/04/rna.1162708.DC2 \\ References This article cites 20 articles, 3 of which can be accessed free at: \\ http://rnajournal.cshlp.org/content/14/10/2115.full.html\#ref-list-1}

\section{License}

Email Alerting Receive free email alerts when new articles cite this article - sign up in the box at the Service top right corner of the article or click here.

To subscribe to RNA go to:

http://rnajournal.cshlp.org/subscriptions 